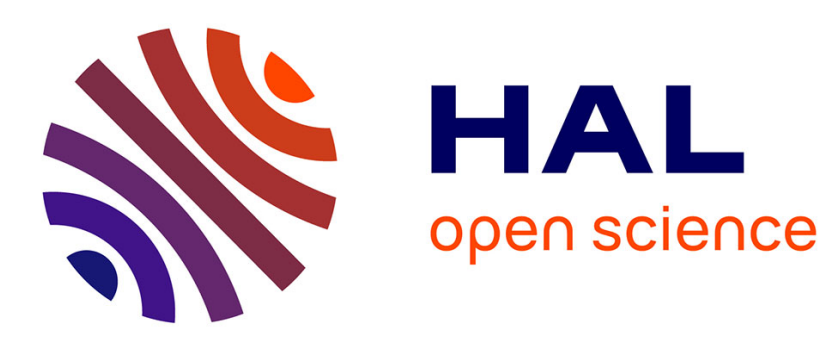

\title{
To pass or not to pass: More a question of body orientation than visual cues
}

Aurore Bourrelly, J-L Vercher, L. Bringoux

\section{To cite this version:}

Aurore Bourrelly, J-L Vercher, L. Bringoux. To pass or not to pass: More a question of body orientation than visual cues. Quarterly Journal of Experimental Psychology, 2014, 67 (9), pp.1668-1681. 10.1080/17470218.2013.864687 . hal-01436019

\section{HAL Id: hal-01436019 https://hal.science/hal-01436019}

Submitted on 2 May 2018

HAL is a multi-disciplinary open access archive for the deposit and dissemination of scientific research documents, whether they are published or not. The documents may come from teaching and research institutions in France or abroad, or from public or private research centers.
L'archive ouverte pluridisciplinaire HAL, est destinée au dépôt et à la diffusion de documents scientifiques de niveau recherche, publiés ou non, émanant des établissements d'enseignement et de recherche français ou étrangers, des laboratoires publics ou privés. 


\title{
To pass or not to pass: More a question of body orientation than visual cues
}

\author{
A. Bourrelly ${ }^{1,2}$, J.-L. Vercher $^{1}$, and L. Bringoux ${ }^{1}$ \\ ${ }^{1}$ Aix-Marseille Université, CNRS, ISM UMR, Marseille, France \\ ${ }^{2}$ Université Lille Nord de France, UVHC, LAMIH-DEMoH, CNRS-UMR, Valenciennes, France
}

This study investigated the influence of pitch body tilt on judging the possibility of passing under high obstacles in the presence of an illusory horizontal self-motion. Seated subjects tilted at various body orientations were asked to estimate the possibility of passing under a projected bar (i.e., a parking barrier), while imagining a forward whole-body displacement normal to gravity. This task was performed under two visual conditions, providing either no visual surroundings or a translational horizontal optic flow that stopped just before the barrier appeared. The results showed a main overestimation of the possibility of passing under the bar in both cases and most importantly revealed a strong influence of body orientation despite the visual specification of horizontal self-motion by optic flow (i.e., both visual conditions yielded a comparable body tilt effect). Specifically, the subjective passability was proportionally deviated towards the body tilt by $46 \%$ of its magnitude when facing a horizontal optic flow and $43 \%$ without visual surroundings. This suggests that the egocentric attraction exerted by body tilt when referring the subjective passability to horizontal self-motion still persists even when anchoring horizontally related visual cues are displayed. These findings are discussed in terms of interaction between spatial references. The link between the reliability of available sensory inputs and the weight attributed to each reference is also addressed.

Keywords: Spatial perception; Body tilt; Vision; Self-motion; Optic flow; Reference frame; Geocentric; Egocentric; Allocentric.

Passing under high obstacles, like the upper part of a door, a tree branch, or a motorway toll height level, is a very natural and successful task daily experienced. In usual situations, such a skill seems easy to perform without any doubts about the effectiveness of action. Nevertheless, in some particular cases, estimating this possibility of action may not be so obvious, leading one to wonder: "Do I pass or not under this obstacle?"

The question raised here concerns the multisensory influence on spatial perceptuomotor skills.
This issue has been addressed from two different, complementary approaches, one focusing on the nature of the information taken from the environment as a consequence of our actions, the other more centred on our capabilities of internally representing the outer world.

From the first perspective, perceptuomotor behaviour when passing under obstacles was previously investigated in a task in which observers walked toward and passed under a horizontal barrier set at different height in front of them

Correspondence should be addressed to A. Bourrelly; L. Bringoux, Aix-Marseille Université, CNRS, ISM UMR 7287, 13288, Marseille Cedex 09, France, Phone: +33 4911722 62, fax: +33 4911722 52, Email: aurore.bourrelly@hotmail.fr; lionel. bringoux@univ-amu.fr

Aurore Bourrelly was supported by a grant from Délégation Générale de l'Armement - Centre National de la Recherche Scientifique [grant number 2007-746]. The authors are grateful to F. Buloup, A. Donneaud, and C. Goulon for technical expertise. 
(Steffanucci \& Geuss, 2010; Van der Meer, 1997; Wagman \& Malek, 2008). In this natural situation, it has been demonstrated that estimating the possibility of passing under high obstacles could rely on the perceptual information provided by the environment itself-that is to say, an affordance, based on a ratio issued from common properties of the subject and the environment (Gibson, 1979; Warren 1984; Warren \& Whang 1987). Within the theoretical framework of affordances, Van der Meer (1997) identified a body-scaled critical height leading to a ducking response, specifically when the barrier elevation is on the amount of 1.04 times the actor's body height for an adult walking at normal speed. A similar body-scaled strategy was also observed without motion, in subjects reporting from a stationary viewpoint the critical height at which they perceived the barrier as "passable" without bending the head (Marcilly \& Luyat, 2008; Steffanucci \& Geuss, 2010; Wagman \& Malek, 2008). In these cases of full vision, the information about passability that is naturally present in the environment is directly perceptible from the structured visual surrounding and does not require any spatial representation. One candidate is the vertical visual direction of motion of the obstacle: "Up" means "passable", "down" means "nonpassable".

From the other perspective, however, under particular conditions, such as in impoverished visual environment, or when displacements toward the obstacle are not directly an option, estimating the possibility of passing under high obstacles may rely on internalized spatial representations that could be crucial for avoiding collision (McIntyre, Zago, Berthoz, \& Lacquaniti, 2001). Particularly, these representations should be defined with respect to specific spatial directions related from the body or gravitational external cues learned through our daily experience. Among them, vertical and horizontal directions represent crucial references that require to be reconstructed, when not directly accessible, in order to anticipate the consequence of actions. For instance, programming a movement for the interception of falling objects was found to require a representation of the direction and dynamics of gravity, even in its absence
(Le Séac'h, Senot, \& McIntyre, 2010). In the matter of the study reported here, questioning the ability to pass under high obstacles in the absence of real motion requires one to figure out a horizontal self-motion (relative to the earth ground). In this context, the effectiveness of the predicted action depends on the ability to represent an accurate horizontal displacement on the basis of available spatially related sensory inputs. Some seminal works have demonstrated that the horizontal direction, when referred to the eyes, is only judged $-2^{\circ}$ lower than the physical reference in darkness (Howard, 1986; MacDougall, 1903). However, recent studies suggested that several environmental factors, such as postural and visual cues, could significantly influence the horizon estimation.

Regarding the effect of postural context, Bringoux, Tamura, Faldon, Gresty, and Bronstein (2004), showed that, in complete darkness, body orientation linearly influenced the subjective horizon in a range from $20^{\circ}$ forward to $30^{\circ}$ backward body tilts. A forward tilt induced a proportional underestimation of horizon height, relative to the physical reference. A comparable linear relationship was subsequently observed when judging objects' elevation and the possibility of passing under them, suggesting that the two estimations shared common processes, intimately linked to the perceived horizontal direction (Bringoux, Robic, Gauthier, \& Vercher, 2008). The origin of this body tilt effect observed in darkness upon judgements of passability was recently investigated, demonstrating that several body parts could jointly intervene in this phenomenon (Bourrelly, Bringoux, \& Vercher, 2009; Bourrelly, Vercher, \& Bringoux, 2011). For instance, we previously highlighted the influence of postural configurations, by manipulating whole-body tilt, trunk tilt alone, and head tilt alone (Bourrelly et al., 2011). Specifically, when compared to the influence of whole-body orientation, the subjective passability was found to be mainly dependent on the orientation of the head $\left(0.77 \mathrm{~cm} \cdot \mathrm{deg}^{-1}\right)$ and trunk $\left(0.27 \mathrm{~cm} \cdot \mathrm{deg}^{-1}\right)$ but not on legs orientation. Overall, these studies addressed the question of how multiple spatial references (i.e., body-related 
or gravity-related) are combined to build a composite reference frame for spatial orientation.

It is also well known that the internally represented horizontal direction is influenced by the static and dynamic visual context. For instance, the perceived horizontal direction has been found to be noticeably deviated towards the pitch orientation of a static tilted visual frame (Matin \& Li, 1992; Stoper \& Cohen, 1989). From a dynamical point of view, other studies demonstrated the influence of the direction of a translational visual motion upon horizontal direction judgements. For instance, $\mathrm{Wu}, \mathrm{He}$, and Ooi (2005) showed that a dynamic visual scene simulating a linear forward motion of the observer could shift the internalized horizontal direction toward the optic flow orientation.

The way postural and visual factors may interact for spatial representation remains nevertheless to be further investigated. Recently, Bourrelly, Vercher, and Bringoux (2010) investigated whether the combination of body tilt and visual cues could impact the perceived direction of a visually induced self-motion. In that case, the direction of the illusory motion, although indicated by visual cues (namely by the focus of expansion of a translational optic flow) has been found to be linearly influenced by body orientation. On the other hand, it has also been reported that vision can fully capture the perception of self-orientation in unusual conflicting situations, such as in the famous "inverted room paradigm" formally investigated by Howard and Templeton (1966) and Jenkin, Dyde, Jenkin, Howard, and Harris (2003).

The question arises as to whether visual cuesnamely, those issued from a radial optic flow inducing a forward-horizontal self-motion-may help reduce or even cancel the formerly observed body tilt influence on the subjective passability under obstacles in darkness (Bourrelly et al., 2011; Bringoux et al., 2008). Specifically, if such visual cues provide relevant information congruent with the task requirement (i.e., estimating a horizontal direction), one could expect a reduced influence of body orientation upon subjective passability. We then tested and compared the ability of passing under obstacles at different body orientations under two visual conditions (without visual surroundings vs. with horizontal optic flow). Besides, findings from this task will help us better understand the interaction between the different systems of coordinates involved in the internal representation of spatial directions.

\section{EXPERIMENTAL STUDY}

\section{Method}

Subjects

Twenty-four subjects with normal or corrected-tonormal vision (by lens correction) gave their informed consent to participate in the study, in compliance with the ethical committee regulating human experimentation in France. Twelve subjects ( 5 males, 7 females; mean age $26.6 \pm 2.0$ years) were tested with no visual surroundings, and 12 other subjects (8 males, 4 females; mean age $29.3 \pm 7.8$ years) were tested with horizontal optic flow. None of them presented a previous history of vestibular and neurological symptoms. All were naive as to the hypothesis under study.

\section{Apparatus}

For each visual condition, subjects were seated on a padded tilting chair allowing body rotations in pitch. They were restrained by means of a shoulder harness with their head strapped and secured on a headrest fixed on the chair. The head was positioned, so that the naso-occipital axis was orthogonal to the direction of gravity when the chair was vertically oriented. The chair was adjusted in height so that the subject's transocular axis coincided with the axis of rotation of the chair. In this way, eye level was kept at the same height with respect to the floor reference $(1.34 \mathrm{~m})$ regardless of the tilt magnitude. Subjects were placed in front of a screen at a distance of $2.28 \mathrm{~m}$. The visual angle of sight was $81^{\circ}$ (horizontal) $\times 48^{\circ}$ (vertical) when binocularly viewed by observers wearing elliptic customized goggles. This ensured that the squared edges of the screen were masked.

A PC Dell Precision 380 computer (Processor: Intel Core i7 950; graphic card: PNY GeForce 
(a)

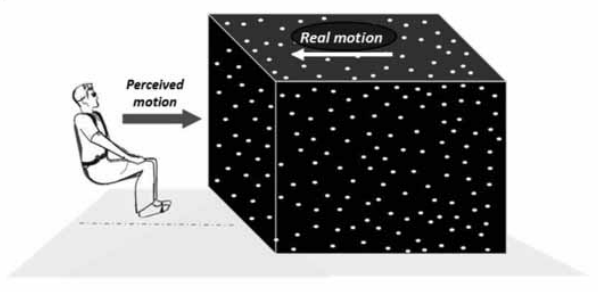

(c)

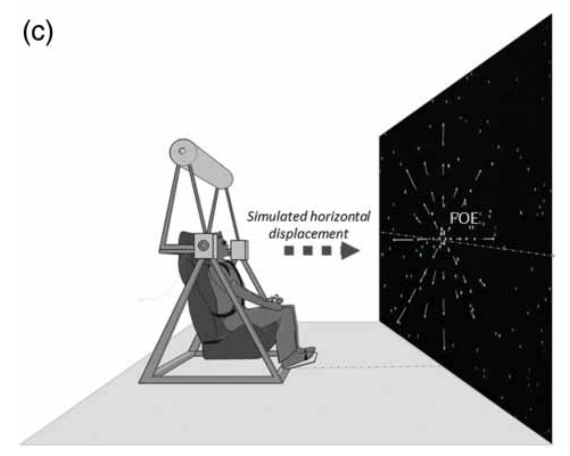

(b)

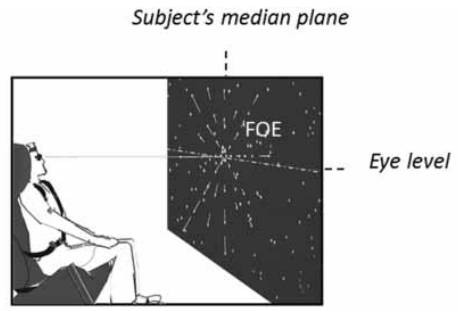

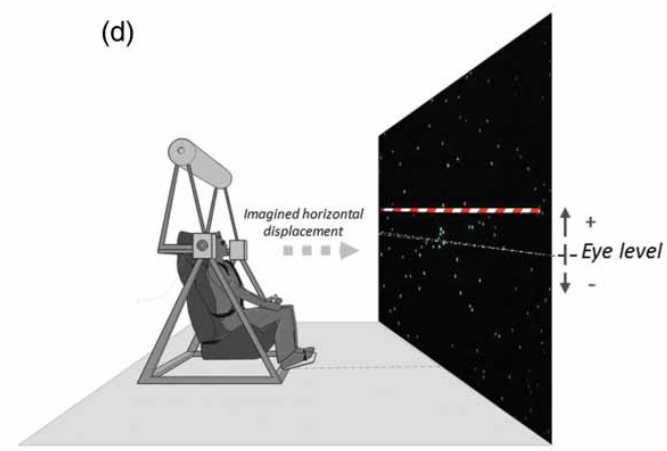

Figure 1. (a) Illustration of the optic flow display presented in a 3D-visual environment. The visual stimulus consisted in an optic flow feld as viewed by an observer translating forward into a $3 D$ cloud of stationary dots. (b) Relative to the stationary observer, the projected stimulus consisted in a cluster of 400 circular dots which radially expanded toward him. The optic flow field was designed to simulate a horizontal displacement. This was obtained by projecting the focus of expansion (FOE), at subjects' eye level, along their median plane. (c) The optic flow was projected for $2 \mathrm{~s}$. (d) At $t=2 \mathrm{~s}$, the optic flow stopped and the visual scene was kept static while a horizontal car park barrier was projected at different heights onto the screen. Subjects had to respond about their possibility of passing under the bar imagining a horizontal displacement.

GTX $5801536 \mathrm{MB}$ ) generated the visual stimulus via our in-house ICE software. A video-projector (refresh rate set to 85 frames/s) projected the visual stimulus onto the screen. In the control condition, the stimulus consisted in a horizontal bar (2 $\mathrm{m}$ long, $5 \mathrm{~cm}$ wide), looking like a parking barrier, projected onto the screen at different heights relative to the subjects' eye level. No visual surroundings were provided in this condition. By contrast, in the horizontal optic flow condition, the visual stimulus was set to simulate an optic flow field viewed by an observer translating forward into a $3 \mathrm{D}$ cloud of 400 stationary dots (diameter $=5$ $\mathrm{mm}$ without local expansion), moved at a constant speed of $66 \mathrm{~m} \mathrm{~s}^{-1}$ [see Bourrelly et al., 2010, for more details; Figure 1(a)]. Relative to the stationary observer, the projected stimulus consisted in a cluster of circular dots, which radially expanded toward him [Figure 1(b) and (c)]. The central focus of expansion of the moving cloud of dots was kept in line with the subjects' eye level, along their median plane [Figure 1(b)]. In this way, the observer experienced a feeling of self-motion in a forward horizontal direction across the visual scene [Figure 1(c)]. The total number of dots was always kept constant on the screen, so that new dots appeared at randomly determined positions in the screen when others went out. When the optic flow stopped, a similar horizontal car park bar was projected onto the screen at different heights relative to the subjects' eye level [Figure 1(d)].

For each condition, subjects were required to estimate whether they could pass under the bar, imagining a forward horizontal displacement. Subjects held in both hands digital response push 
buttons for judgement settings. Responses were recorded by using the ADwin-Gold system (KeithleyC) piloted via our in-house Docometer software. Throughout the experiment, subjects were placed in darkness without any other external visual cue than the visual scene projected onto the screen.

\section{Procedure}

Nine angles of body tilt $\left(0^{\circ} ;-5^{\circ},-10^{\circ},-15^{\circ},-20^{\circ}\right.$ forward; and $+5^{\circ},+10^{\circ},+15^{\circ},+20^{\circ}$ backward relative to gravity) were manipulated for each visual condition. For each body orientation, 18 bar elevations $(0 ;+2.5,+5,+7.5,+10,+12.5$, $+15,+17.5,+20,+25 \mathrm{~cm}$ upward elevations; and $-2.5,-5,-7.5,-10,-12.5,-15,-17.5$, $-20 \mathrm{~cm}$ downward elevations from eye level; i.e., respectively, $0, \pm 1.2^{\circ}, \pm 2.4^{\circ}, \pm 3.5^{\circ}, \pm 4.7^{\circ}$, $\pm 5.9^{\circ}, \pm 7^{\circ}, \pm 8.2^{\circ}, \pm 9.6^{\circ}$, and $+11.6^{\circ}$ elevations from eye level) were randomly presented to the subjects. Before the lights were turned off, subjects were required to attentively consider the distance that separated them from the screen. A first presentation of the car park bar was given to the subjects in order to help them to evaluate its width and its distance of projection (kept constant across the trials).Subjects were asked to answer the following question: "Do you think you could pass under the bar, in the present body orientation, imagining a forward horizontal displacement of your body?".

Subjects were first positioned at the desired body angle relative to gravity in complete darkness. The chair was rotated at constant velocity during $11 \mathrm{~s}$, with a period of initial acceleration and final deceleration of $2 \mathrm{~s}\left(0.4^{\circ} \cdot \mathrm{s}^{-1}\right.$ and $0.2^{\circ} \cdot \mathrm{s}^{-2}$ for $\pm 5^{\circ}$ tilt, $0.8^{\circ} \cdot \mathrm{s}^{-1}$ and $0.4^{\circ} \cdot \mathrm{s}^{-2}$ for $\pm 10^{\circ}$ tilt, $1.2^{\circ} \cdot \mathrm{s}^{-1}$ and $0.6^{\circ} \cdot \mathrm{s}^{-2}$ for $\pm 15^{\circ}$ tilt, $1.6^{\circ} \cdot \mathrm{s}^{-1}$ and $0.8^{\circ} \cdot \mathrm{s}^{-2}$ for $\pm 20^{\circ}$ tilt). This was followed by $15 \mathrm{~s}$ of rest. This specific duration was chosen as a compromise between the weakest vestibular resting discharge allowing consideration of postrotational effects as negligible (Benson, 1990; Goldberg \& Fernandez, 1977) and limited somatosensory adaptation due to the subsequent period of static tilt (Higashiyama \& Koga, 1998).

Stationary subjects were then asked to open their eyes and to observe the visual scene that was projected in front of them. In the control condition, subjects were instructed to gaze at the horizontal bar, which appeared on the screen for $4 \mathrm{~s}$. Then, the bar was switched off, and subjects were asked to respond about the possibility of passing under the bar, via a forced-choice judgement by means of two hand-held buttons. In the horizontal optic flow condition, subjects were asked to observe the projected optic flow for $2 \mathrm{~s}$. During this phase, in which all participants reported having experienced a clear feeling of forward self-motion, subjects were required to orient their gaze in the direction toward which they felt they were translating. Then, the visual scene was frozen, and the horizontal bar appeared on the screen for $0.3 \mathrm{~s}$ while the subjects were required to gaze at the bar (the delay of saccadic eye movements being $200 \mathrm{~ms}+$ $85 \mathrm{~ms}$ of decision process, one can easily consider that less than $300 \mathrm{~ms}$ was sufficient for the subjects to orient their gaze toward the bar; Robinson, 1973). The optic flow was stopped before the bar appeared in order to avoid the observers referring to purely visual allocentric coding-namely, judging passability by a direct comparison between central focus of expansion and bar elevation. Then, the visual scene disappeared, and subjects were asked to respond about the possibility of passing under the bar, using the same forcedchoice judgement. Judgement settings were recorded via the ADwin-Pro system (KeithleyC) piloted via our in-house software (Docometre). None of the subjects reported any difficulty in following the task requirements. At the end of the sequence, the chair was brought back to the vertical, and the room lights were turned on for $5 \mathrm{~s}$ before a new sequence was launched. For a given body orientation, each visual stimulus was repeated three times in a pseudorandomized order. This order was strictly counterbalanced for half of the subjects. Finally, the total number of judgements was 486 $(3 \times 18 \times 9)$ for a total session duration of $60 \mathrm{~min}$ per visual condition. The instructions were frequently repeated to keep subjects alert and concentrating on the task throughout the experiment. During the experiment, subjects were not informed about the motion direction simulated by optic flow or about the number and height of bar elevations. 

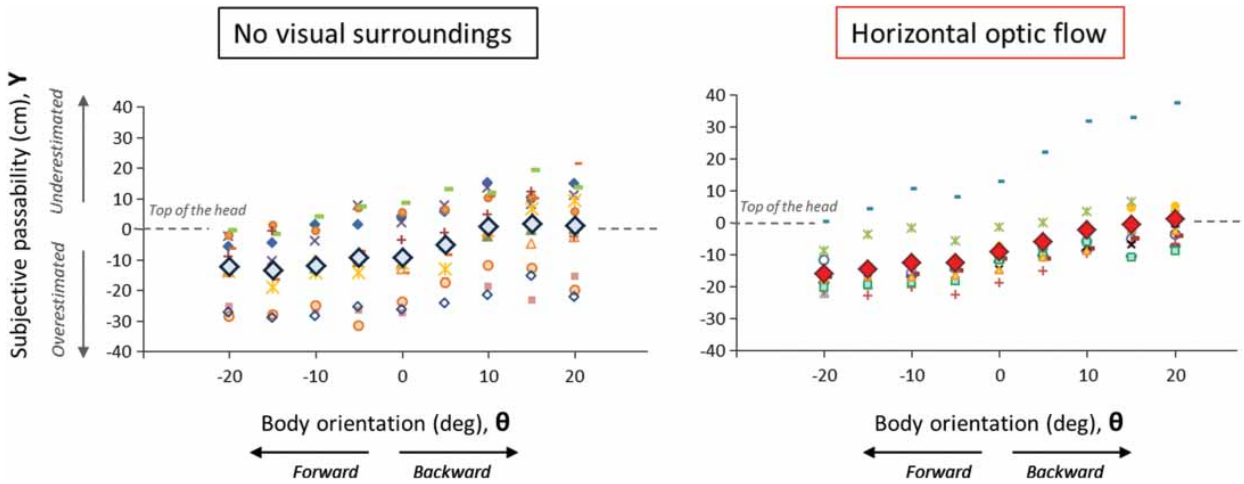

Figure 2. Individual subjective passability and mean subjective passability ( ) relative to the top of the head obtained for different body orientations, in both visual conditions, with no visual surroundings and with horizontal optic flow. The figure shows comparable individual profiles although individual differences in the offsets.

\section{Data processing}

For each visual condition, judgement settings were first converted into binary values. For each bar elevation, a score of 1 was attributed when subjects thought they could pass under the bar, and a score of 0 was attributed when subjects responded they could not pass under the bar. A subsequent "probit" model, using a nonlinear regression analysis for dichotomic variables, was achieved on the binary responses obtained for each body orientation in order to determine for each bar height the probability $p$ that subjects estimated they could pass under the bar. This permitted us to mathematically determine an indirect variable - that is, the subjective passability, corresponding to the minimal subjective height (in $\mathrm{cm}$ ) relative to eye level at which subjects estimated they could pass under the bar $(p=50 \%)$. The slope of the tangent at the inflection point of the probit curve gave an indication about the discrimination sensitivity of the socalled subjective passability relative to the chosen increments; the sharper the slope, the higher the discrimination sensitivity. An analysis of variance (ANOVA) with repeated measures was performed on the slopes of the probit function to ensure there was no difference in the discrimination sensitivity whatever the experimental condition. Judgements of "subjective passability", initially referred to eye level for convenience, were subsequently reported to the top of the head, defined as the highest physical point of the head from the horizontal floor of the room measured for each subject in each body orientation. Hence, data were expressed in term of vertical deviation (or error, in $\mathrm{cm}$ ) relative to the top of the head (that is, the true level of passability). Positive values corresponded to an overestimation of the possibility of passing under obstacles relative to the top of the head, and negative values corresponded to an underestimation of the possibility of passing under obstacles (Figure 2).

Preliminary analyses were conducted on the variance distribution (i.e., Levene's test assessing variance homogeneity for both groups of subjects as well as direct comparison of intrasubject variances between groups and body orientations). As no difference was found on this parameter, we were allowed to subsequently perform mean comparisons of the subjective passability observed in all the experimental situations.

To that aim, a 2 (visual condition: with no visual surroundings and with horizontal optic flow) $\times 9$ (body orientation: $0^{\circ} ;-5^{\circ},-10^{\circ},-15^{\circ},-20^{\circ}$ forward; and $+5^{\circ},+10^{\circ},+15^{\circ},+20^{\circ}$ backward relative to gravity) ANOVA with repeated measures on the last factor was conducted on the subjective passability calculated for each subject. A linear regression analysis was then applied to the data to characterize the type of influence exerted by body orientation for each visual condition. Finally, differences between visual conditions were also tested by comparing the 
Table 1. The main body orientation effect

\begin{tabular}{|c|c|c|c|c|c|c|c|c|c|}
\hline \multirow[b]{2}{*}{ Body orientation } & \multicolumn{9}{|c|}{ Body orientation } \\
\hline & $-20^{\circ}$ & $-15^{\circ}$ & $-10^{\circ}$ & $-5^{\circ}$ & $0^{\circ}$ & $5^{\circ}$ & $10^{\circ}$ & $15^{\circ}$ & $20^{\circ}$ \\
\hline$-20^{\circ}$ & - & $n s$ & ns & $*$ & $* * *$ & $* * *$ & $* * *$ & $* * *$ & $* * *$ \\
\hline$-15^{\circ}$ & & - & $n s$ & $*$ & $* * *$ & $* * * *$ & $* * * *$ & $* * *$ & $* * *$ \\
\hline$-10^{\circ}$ & & & - & $n s$ & $*$ & $* * *$ & $* * *$ & $* * *$ & $* * *$ \\
\hline$-5^{\circ}$ & & & & - & $n s$ & $* * *$ & $* * *$ & $* * *$ & $* * * *$ \\
\hline $0^{\circ}$ & & & & & - & $* *$ & $* * *$ & $* * *$ & $* * * *$ \\
\hline $5^{\circ}$ & & & & & & - & $* * * *$ & $* * *$ & $* * *$ \\
\hline $10^{\circ}$ & & & & & & & - & $n s$ & $n s$ \\
\hline $15^{\circ}$ & & & & & & & & - & ns \\
\hline $20^{\circ}$ & & & & & & & & & - \\
\hline
\end{tabular}

Note: Summary table of the Newman-Keuls pairwise comparisons for the mean estimates of subjective passability. ${ }^{*} p<.05 .{ }^{* *} p<.01 .{ }^{* * *} p<.001 . n s=$ no significant difference.

slopes of the regression lines obtained for each subject in each condition ( $t$-test for independent samples).

\section{Results}

\section{Probit analysis}

A nonlinear regression analysis (probit function) was performed on the binary values to determine the subjective passability for each subject in each experimental condition (see "Method"). To assess

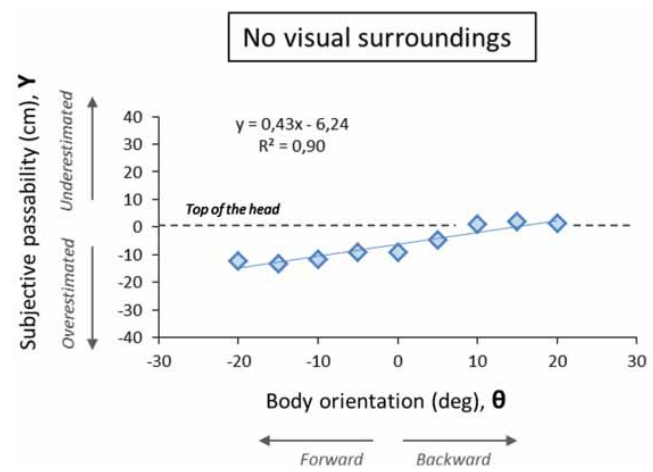

the discrimination sensibility of the probit processing, a one-way ANOVA with repeated measures was performed on the slopes calculated at the inflection point of the probit function curve for each body orientation. Results showed there was no significant difference in the discrimination sensitivity of the subjective passability, whatever the body orientation in the control condition, $F(8$, $88)=1.14 ; p=.34$, as well as in the horizontal optic flow condition, $F(8,88)=1.18 ; p=.32$.

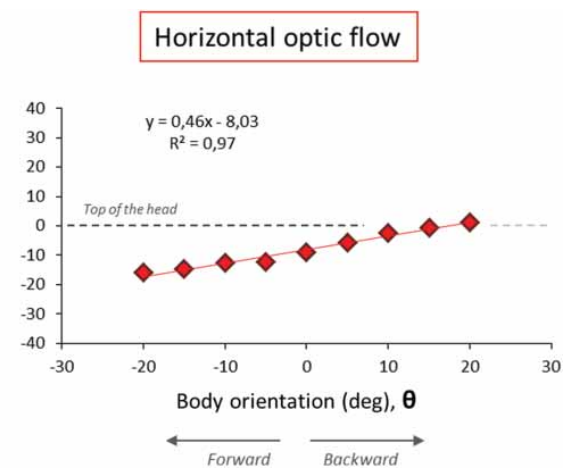

Figure 3. Linear regression applied to the mean estimates of the subjective passability under obstacles relative to the angle of tilt with no visual surrounding, and with horizontal optic flow. The more the subjects were tilted forward (up to-20-deg), the lower the thresholds, that is, the more the subjects overestimated the obstacle elevation and their capacity of passing under. The equation of the regression line shows an influence of body orientation about $43 \%$ and $46 \%$, respectively, on the subjective estimates. The coefficients -6.24 and -8.03 show a general lowering of the subjective passability relative to the top of the head, that is the true level of passability, at $0^{\circ}$ of pitch body orientation. Overall, negative values indicated that the possibility of passing under the bar was overestimated. $R^{2}$ provides a measure of how well the recorded data are likely to be predicted by the linear statistical model, $\left(^{* * * *}=p<.001\right)$. Note that the effect of body tilt upon subjective passability is fully comparable in both visual conditions ( $43 \%$ and $46 \%$ ). 
Table 2. Results of the linear regression analysis between the mean subjective "passability" and the angle of tilt

\begin{tabular}{lccc}
\hline Experimental conditions & Equation of the regression lines & $\mathrm{R}^{2}$ & $\mathrm{p}$ \\
\hline No visual surroundings & $y=0.43 \theta-6.24$ & .90 & $<.001$ \\
Horizontal optic flow & $y=0.46 \theta-8.03$ & .97 & $<.001$ \\
\hline
\end{tabular}

Note: Mean subjective "passability" in $\mathrm{cm}$. Angle of tilt in degrees.

\section{Mean comparisons of subjective passability}

The 2 (visual condition) $\times 9$ (body orientation) ANOVA conducted on the mean estimates of subjective passability revealed a significant effect of body orientation, $F(8,178)=62.03, p<.001$, $1-\beta=1$ (Figure 2). By contrast, no difference was found between the two visual conditions, $F(1,22)=0.20, p=.66 ; 1-\beta=.07$, and no significant interaction was found between the visual condition and body orientation, $F(8,176)=0.78$, $p<.62,1-\beta=.36$. This means that the subjective passability in complete darkness did not differ from estimates obtained with horizontal optic flow. In other words, the main effect of body orientation upon judgements was not affected by the visual condition. Post hoc analyses (NewmanKeuls test) are reported in Table 1.

\section{Linear regression analysis}

A linear regression analysis was applied to the individual estimates of subjective passability in order to characterize the influence of body orientation upon the judgements (Figure 3). Results highlighted a significant linear effect of body tilt on the subjective passability in both visual conditions $[F(1,106)=$ 47.22, $p<.001,1-\beta=.99$ with no visual surroundings, and $F(1,106)=23.12, p<.001,1-$ $\beta=.99$ with horizontal optic flow]. Specifically, the more the subjects were tilted forward, the more they underestimated the possibility of passing under a given obstacle. The equations of the regression lines calculated on the mean subjective estimates are summarized in Table 2.

The equations of the regression lines were about $y=a \theta+b$, where " $y$ " corresponds to the subjective passability, " $a$ " to the weight of the body orientation influence in $\mathrm{cm} \cdot \mathrm{deg}^{-1}$, " $\theta$ " to the body orientation angle, and " $b$ " to the offset of the regression line, here characterizing the general lowering of the subjective estimates relative to the top of the headthat is, the true level of passability. Results showed a comparable linear influence of body orientation whatever the visual condition $(0.43$ $\mathrm{cm} \cdot \mathrm{deg}^{-1}$ and $0.46 \mathrm{~cm} \cdot \mathrm{deg}^{-1}$, with no visual surroundings and with horizontal optic flow, respectively) as well as comparable negative offsets in both judgements $(-6.24$ and -8.03 , respectively).

In order to compare the linear influences between the two visual conditions, a $t$-test for independent samples was conducted on the slope coefficients derived from the individual regression lines obtained for each participant in each visual condition. Results did not reveal any significant differences between visual conditions $(t=0.37 ; p=.71)$.

\section{Discussion}

The present study investigated whether orientational visual cues from optic flow may help to reduce the influence of body tilt previously observed in darkness on estimating the passability under a barrier when imagining a forward horizontal displacement (Bourrelly et al., 2009, 2011; Bringoux et al., 2008). The underlying issues were to better understand how spatial references could interact for the perception of space.

The main finding of this study was that the subjective passability was significantly affected by the angle of body tilt, even when the horizontal direction of displacement was clearly specified by optic flow. The relative influence of visual and postural cues on this spatial task are first discussed, before considering the way in which the different available spatial cues may interact and lead to a unified perception of space. 
Visual versus postural information for estimating the passability under obstacles

As previously observed by Bourrelly et al. (2009), our results showed that the mean subjective passability is overall lower than the physical minimal height required to achieve the task $(-6.24 \mathrm{~cm}$ and $-8.03 \mathrm{~cm}$ with no visual surroundings and with horizontal optic flow, respectively). These results highlighted a global overestimation of the possibility of passing under obstacles (i.e., typically, subjects estimated they were able to pass under obstacles that were actually located below the top of their head; Bourrelly et al., 2009; Bringoux et al., 2008). This phenomenon may be related to the $30-\mathrm{deg}$ backward orientation of the saccular and utricular maculae relative to the head (Rosenhall, 1972). Indeed, as proposed in particular by Bortolami, Pierobon, DiZio, and Lackner (2006), this tilt may cause a bias (zero shift and backward-forward asymmetry) in the vestibular signal, when the head is positioned in a zero-tilt posture (we used the naso-occipital axis as a reference for horizontality). As a consequence, obstacles whose elevation is referred to the subjective visual horizon will be considered higher than they actually are, since the head is perceived as more tilted than it actually is.

The core result of the present study is that the substantial effect of body tilt on estimating the possibility of passing under high obstacles was not attenuated by directional cues issued from optic flow. We still found indeed a proportional influence of body orientation on the judgements in a \pm 20 deg range. Specifically, the more the body was oriented downward, the more the possibility of passing under high obstacles was overestimated. Conversely, this overestimation was reduced when the body was oriented backward. Most importantly, the effect of body tilt upon subjective passability is comparable for a similar task but performed in complete darkness ( $46 \%$ vs. $43 \%$, respectively). Then, it is obvious from the present data that the subjective passability was attracted toward a bodyrelated direction despite the fact that the horizontal direction of self-motion was visually specified by optic flow.

As a whole, these findings support a greater influence of postural cues (e.g., idiotropic,
Mittelstaedt, 1992) relative to visual cues than has usually been reported for other common spatial judgement tasks. Indeed, most of the previous studies manipulating postural and/or visual context when asking for the subjective visual or postural vertical (Barnett-Cowan \& Harris, 2008; Howard \& Childerson, 1994) or the visual perceived eye level (Li, Dallal, \& Matin, 2001) emphasized the prominent role of vision upon tilt. Several hypotheses may be advanced to explain this apparent discrepancy, mostly related to the nature of visual information. Considering the present results, it could be suggested that the structure of the projected optic flow is not rich enough to accurately specify self-motion direction while the body is tilted. However, the unambiguous feeling of self-motion reported here by the subjects is a strong support for considering the visual flow as relevant to generate vection. One may also hypothesize that additional information about external space through more natural and meaningful visual scenes could increase the influence of vision upon judgements (Bringoux et al., 2009). For instance, adding a fixed 3D frame surrounding the dynamic visual scene could enhance the anchoring role of visual cues and, incidentally, diminish the body tilt attraction found in the present experiment. Still, to our knowledge, this study is the first to report such a remaining and still consistent effect of postural orientation in a spatial task where visuospatial cues are otherwise available.

\section{Weighting spatial references into a "composite" reference frame?}

If, as noticed above, postural orientation has been found to strongly influence the subjective passability, one has also to consider the non-negligible role of visual and/or gravity-related cues in judgement making. Indeed, perceptual responses were not $100 \%$ dependent upon postural orientation (as shown by the regression line coefficient), and the remaining influence of gravitational or/and visual cues raises the issue of how this information is combined to yield a unified perception of space.

We assume here that available sensory cues in the present task convey spatial information about salient directions of space, called references 
(Howard, 1982). Among them, we consider egocentric references (i.e., body-related axes such as the head-referenced eye level or the $z$-longitudinal body axis), allocentric references (i.e., salient directions from the surroundings such as the perspective lines of a room or given by the orientation of the support surface), and geocentric references (i.e., anchoring earth-based directions, such as the direction of gravity and the physical horizon). In usual situations, these spatial references naturally matched, some of them being issued from different sensory inputs (e.g., gravity sensing by vestibular and somatosensory cues), whereas others were sometimes conveyed by single sensory inputs (e. g., up-down direction of the surroundings mediated by visual cues only). In this context, estimating the spatial location of an object, for instance the height of an obstacle, can be done by using any of the available references. However, when these references are noncongruent (i.e., spatial conflicting situation), the question arises as to how these spatial cues are processed and integrated by the central nervous system (CNS).

Here, we propose to extend the recent views explaining multisensory integration process (i.e., probabilistic approaches based on Bayesian models; Ernst \& Banks, 2002; Vingerhoets, De Vrijer, Van Gisbergen, \& Medendorp, 2009) at the level of spatial references. According to this, we hypothesize that the CNS may attribute different weights to the available spatial references, not only depending on their reliability (see the Appendix supporting a limited predictive power of a simple model based on signals reliability), but also on previous spatial experience and expectations in order to build a coherent perceptual space. Specifically, during the integration process, if the CNS may likely take into account the signal-to-noise ratio giving access to a spatial reference (sensory reliability), it may also assimilate priors affecting the way the reference is a priori regarded (sensory relevance).

In this framework, our data clearly support the idea of a substantial weight attributed to some egocentric references when the reliability/relevance of allocentric and geocentric references is low. Specifically, it is obvious in our task that the visually defined direction of self-motion, which specified an allocentric reference of horizontality, might not be very salient since it had to be extracted from optic flow. Furthermore, the gravity-related cues giving access to the geocentric reference of horizontality (through vestibular and somatosensory inputs) have been found rather imprecise (when considering nonrefreshed vestibular graviceptive inputs; Bringoux et al., 2004) or subject to adaptation (because of the progressive decay of the somatosensory information coding static touch and pressure; Higashiyama \& Koga, 1998). On the other hand, as it is rather usual to experience forward motion along a sagittal head axis (Pozzo, Papaxanthis, Stapley, \& Berthoz, 1998), head-referenced eye level may be naturally considered as an egocentric reference for horizontality (Stoper \& Cohen, 1989) and may be substantially weighted by the CNS receiving otherwise ambiguous spatial information. If head-referenced eye level is a good candidate to become here a key reference for horizontal estimation of self-motion used for judgements of passability, its weight seems to remain unchanged whatever the body tilt (in the range of the tested angles). Indeed, the linear regression analysis attested to a similar and constant influence of the egocentric reference across various body orientations. We suggest here that the weight of this constant influence can be directly obtained from the regression line coefficient (i.e., $0.46 \mathrm{~cm} . \mathrm{deg}^{-1}$ for the horizontal optic flow condition).

Following our main interpretation, and assuming that an internal representation of space is required to perform the task when facing conflicting and/or impoverished sensory environments, we hypothesize that a unique "composite" reference frame could emerge from the weighted combination of the available spatial references (Bringoux et al., 2008; Gueguen, Vuillerme, \& Isableu, 2012; Luyat, Mobarek, Leconte, \& Gentaz, 2005). This view is somehow different from the more classical ones supporting that several reference frames could be specifically elaborated at a representational level (Batista, 2002; Brotchie, Andersen, Snyder, \& Goodman, 1995; Ghafouri, Archambault, Adamovich, \& Feldman, 2002; Snyder, Grieve, Brotchie, \& Andersen, 1998). Indeed, if some previous works have 
suggested that spatial reference frames may be identified as preexistent neurophysiological structures, exclusive from one to others (Galati et al., 2000; Snyder et al., 1998), some recent studies argue for less distinctive loci of spatial representations, often overlapped and differently activated depending on the task constraints (Committeri et al., 2004; Lopez, Lacour, \& Borel, 2005). Hence, composite representations may arise from the combination between several spatial references. Further research is needed to explore the way a subjective reference frame may emerge, adapt, and transfer across different spatial tasks. Computational approaches could be a powerful tool for predicting perceptual consequences associated to the combination of redundant or concurrent spatial inputs. A preliminary step to initiate such an approach is presented in the Appendix.

\section{Conclusion}

Overall, this study strongly supports the hypothesis of a substantial egocentric influence on subjective passability as a powerful phenomenon resistant to the presentation of horizontal cues in the visual scene. These findings could be particularly relevant in the context of aeronautics where pilots, oriented $30^{\circ}$ backward (Roumes \& Grau, 2003) may have to judge earth-based directions of space under poor visual conditions (under fog or darkness). This study may also be of value for further research on multisensory implication in space perception. For example, it could be particularly interesting to question the contribution of specific sensory cues in the reweighting processes affecting the combination between available spatial references. In this vein, the changes observed in the weight of a specific spatial reference over time could be highly informative to better understand how these spatial cues dynamically interact (Bringoux et al., 2009; Scotto Di Cesare, Bringoux, Bourdin, Sarlegna, \& Mestre, 2011).

Original manuscript received 13 October 2012 Accepted revision received 27 October 2013 First published online 14 January 2014

\section{REFERENCES}

Barnett-Cowan, M., \& Harris, L. R. (2008). Perceived self-orientation in allocentric and egocentric space: Effects of visual and physical tilt on saccadic and tactile measures. Brain Research, 1242, 231-243. doi:10.1016/j.brainres.2008.07.075

Batista, A. (2002). Inner space: Reference frames. Current Biology, 12, 380-383. doi:10.1016/S09609822(02)00878-3

Benson, A. J. (1990). Sensor functions and limitations of the vestibular system. In R. Warren \& A. H. Wertheim (Eds.), Perception and control of selfmotion (pp. 145-170). Hillsdale, US: Lawrence Erlbaum Associates.

Bonneh, Y. S., Cooperman, A., \& Sagi, D. (2001). Motion-induced blindness in normal observers. Nature, 411, 798-801. doi:10.1038/35081073

Bortolami, S. B., Pierobon, A., DiZio, P., \& Lackner, J. R. (2006). Localization of the subjective vertical during roll, pitch, and recumbent yaw body tilt. Experimental Brain Research, 173, 364-373. doi:10. 1007/s00221-006-0385-y

Bourrelly, A., Bringoux, L., \& Vercher, J. L. (2009). Influence of gaze elevation on estimating the possibility of passing under high obstacles during body tilt. Experimental Brain Research, 193, 19-28. doi:10.1007/s00221-008-1589-0

Bourrelly, A., Vercher, J. L., \& Bringoux, L. (2010). Pitch body orientation influences the perception of self-motion direction induced by optic flow. Neuroscience Letters, 482, 193-197. doi:10.1016/j. neulet.2010.07.028

Bourrelly, A., Vercher, J. L., \& Bringoux, L. (2011). Postural configuration affects the perception of earth-based space during pitch tilt. Acta Psychologica, 138, 119-125. doi:10.1016/j.actpsy.2011.05.014

Bringoux, L., Bourdin, C., Lepecq, J. C., Sandor, P. M., Pergandi, J. M., \& Mestre, D. (2009). Interaction between reference frames during subjective vertical estimates in a tilted immersive virtual environment. Perception, 38, 1053-1071. doi:10.1068/p6089

Bringoux, L., Robic, G., Gauthier, G. M., \& Vercher, J. L. (2008). Judging beforehand the possibility of passing under obstacles without motion: The influence of egocentric and geocentric frames of reference. Experimental Brain Research, 185, 673-680. doi:10. 1007/s00221-007-1194-7

Bringoux, L., Tamura, K., Faldon, M., Gresty, M. A., \& Bronstein, A. M. (2004). Influence of whole-body pitch tilt and kinesthetic cues on the perceived 
gravity-referenced eye level. Experimental Brain Research, 155, 385-392. doi:10.1007/s00221-0031742-8

Brotchie, P. R., Andersen, R. A., Snyder, L. H., \& Goodman, S. J. (1995). Head position signals used by parietal neurons to encode locations of visual stimuli. Nature, 375, 232-235. doi:10.1038/ 375232a0

Committeri, G., Galati, G., Paradis, A. L., Pizzamiglio, L., Berthoz, A., \& Le Bihan, D. (2004). Reference frames for spatial cognition: Different brain areas are involved in viewer-, object-, and landmark-centered judgments about object location. Journal of Cognitive Neuroscience, 16, 1517-1535. doi:10.1162/ 0898929042568550

Ernst, M. O., Banks, M. S. (2002). Humans integrate visual and haptic information in a statistically optimal fashion. Nature, 415, 429-433. doi:10. 1038/415429a

Galati, G., Lobel, E., Vallard, G., Berthoz, A., Pizzamiglio, L., \& Le Bihan, D. (2000). The neural basis of egocentric and allocentric coding of space in human: A functional magnetic resonance study. Experimental Brain Research, 133, 156-164. doi:10. 1007/s002210000375

Ghafouri, M., Archambault, P. S., Adamovich, S. V., \& Feldman, A. G. (2002). Pointing movements may be produced in different frames of reference depending on the task demand. Brain Research, 929, 117-128. doi:10.1016/S0006-8993(01)03332-7

Gibson, J. J. (1979). The ecological approach to visual perception. Boston, US: Houghton Mifflin.

Goldberg, J. M., \& Fernandez, C. (1977). Conduction times and background discharge of vestibular afferents. Brain Research, 122, 545-550. doi:10.1016/ 0006-8993(77)90465-6

Gueguen, M., Vuillerme, N., \& Isableu, B. (2012). Does the integration of haptic and visual cues reduce the effect of a biased visual reference frame on the subjective head orientation?. PLoS ONE, 7, 1-14, e34380. doi:10.1371/journal.pone.0034380

Higashiyama, A., \& Koga, K. (1998). Apparent body tilt and postural aftereffect. Perception E Psychophysics, 60, 331-347. doi:10.3758/BF03206041

Howard, I. P. (1982). Human visual orientation. NewYork, US: John Wiley.

Howard, I. P. (1986). The perception of posture, self motion, and the visual vertical. In K. R. Boff, L. Kaufman, \&J. P. Thomas (Eds.), Handbook of perception and human performance (Vol. 1, Chapter. 18, pp. 1-62). New York: JohnWiley.
Howard, I. P., \& Childerson, L. (1994). The contribution of motion, the visual frame, and visual polarity to sensations of body tilt. Perception, 23, 753-762. doi:10.1068/p230753

Howard, I. P., \& Templeton, W. B. (1966). Human Spatial Perception. New York: John Wiley.

Jenkin, H. L., Dyde, R. T., Jenkin, M. R., Howard, I. P., \& Harris, L. R. (2003). Relative role of visual and non-visual cues in determining the direction of 'up': Experiments in the York tilted room facility. Journal of Vestibular Research, 13, 287-293. doi:10.1016/j. actaastro.2005.01.030

Le Séac'h, A., Senot, P., \& McIntyre, J. (2010). Egocentric and allocentric reference frames for catching a falling object. Experimental Brain Research, 201, 653-662. doi:10.1007/s00221-009-2081-1

Li, W., Dallal, N., \& Matin, L. (2001). Influences of visual pitch and visual yaw on visually perceived eye level (VPEL) and straight ahead (VPSA) for erect and rolled-to-horizontal observers. Vision Research, 41, 2873-2894. doi:10.1016/S0042-6989(01)00165-1

Lopez, C., Lacour, M., \& Borel, L. (2005). Perception de la verticalité et représentation spatiale dans les aires corticales vestibulaires. In M. Lacour \& B. Weber (Eds.), Bipédie, contrôle postural et représentation corticale (pp. 35-86). Marseille, France: Solal.

Luyat, M., Mobarek, S., Leconte, C., \& Gentaz, E. (2005). The plasticity of gravitational reference frame and the subjective vertical: Peripheral visual information affects the oblique effect. Neuroscience Letters, 385, 215-219. doi:10.1016/j.neulet.2005.05.044

MacDougall, R. (1903). The subjective horizon. Psychological Review, 4, 145-166. psycnet.apa.org/ psycinfo/1926-02633-001

Marcilly, R., \& Luyat, M. (2008). The role of eye height in judgment of an affordance of passage under a barrier. Current Psychology Letters, 24, 12-24. URL: cpl.revues.org/document3443.html

Matin, L., \& Li, W. (1992). Visually perceived eye level: Changes induced by a pitched from -vertical 2-line visual field. Journal of Experimental Psychology: Human Perception E Performance, 18, 257-289. doi:10.1037/0096-1523.18.1.257

McIntyre, J., Zago, M., Berthoz, A., \& Lacquaniti, F. (2001). Does the brain model Newton's laws? Nature Neuroscience, 4, 693-694. doi:10.1038/89477

van der Meer, A. L. H. (1997). Visual guidance of passing under a barrier. Early Development \& Parenting, 6, 149-157. doi:10.1002/(SICI)1099-0917(199709/12) 6:3/4\&lt;149::AID-EDP154>3.0.CO;2-2 
Mittelstaedt, H. (1992). Somatic versus vestibular gravity reception in man. Annals of the New York Academy of Sciences, 656, 124-139. doi:10.1111/j.1749-6632. 1992.tb25204.x

Pozzo, T., Papaxanthis, C., Stapley, P., \& Berthoz, A. (1998). The sensorimotor and cognitive integration of gravity. Brain Research Review, 28, 92-101. doi:10.1016/S0165-0173(98)00030-7

Robinson, D. A. (1973). Models of the saccadic eye movement control system. Biological Cybernetics, 14, 71-83. doi:10.1007/BF00288906

Rosenhall, U. (1972). Vestibular macular mapping in man. Annals of Otology, Rhinology \& Laryngology, 81, 339-351.

Roumes, C., \& Grau, J. Y. (2003). Rafale: A human factors view of the man machine interface. Médecine aéronautique et spatiale, 45, 23-26.

Scotto Di Cesare, C., Bringoux, L., Bourdin, C., Sarlegna, F., \& Mestre, D. (2011). Spatial localization investigated by continuous pointing during visual and gravitoinertial changes. Experimental Brain Research, 215, 173-182. doi:10.1007/s00221-011-2884-8

Snyder, L. H., Grieve, K. L., Brotchie, P., \& Andersen, R. A. (1998). Separate body- and world-referenced representations of visual space in parietal cortex. Nature, 394, 887-891. doi:10.1038/29777

Steffanucci, J. K., \& Geuss, N. M. (2010). Duck! Scaling the height of a horizontal barrier to body height. Attention, Perception, E Psychophysics, 72, 13381349. doi:10/3758/APP.72.5.1338

Stoper, A. E., \& Cohen, M. M. (1989). Effect of structured visual environments on apparent eye level.

\section{APPENDIX}

\section{A computational model based on maximum- likelihood estimation}

A complementary support to discuss our results lies in the conceptual framework of computational models (Bayesian model; Tagliabue \& McIntyre, 2011). Maximum-likelihood estimation (MLE) is indeed a method for estimating the parameters of a statistical model. When applied to a data set, MLE provides estimates for the model's parameters based on signal reliability. The latter, which is defined by the inverse of the response variability associated to a given input, may be considered as the weight attributed to this input. In the present section, we developed a complementary analysis relying on Bayesian rules for testing how subjective passability, when combined visual and body-related cues are available, could be predicted from respective unimodal conditions.
Perception E Psychophysics, 46, 469-475. doi:10. 3758/BF03210862

Tagliabue, M., \& McIntyre, J. (2011). Necessity is the mother of invention: reconstructing missing sensory information in multiple, concurrent reference frames for eye-hand coordination. The Journal of Neuroscience, 31, 1397-409. doi:10.1523/ JNEUROSCI.0623-10.2011

Vingerhoets, R. A. A., De Vrijer, M., Van Gisbergen, J. A. M., \& Medendorp, W. P. (2009). Fusion of visual and vestibular tilt cues in the perception of visual vertical. Journal of Neurophysiology, 101, 13211333. doi:10.1152/jn. 90725.2008

Wagman, J. B., \& Malek, E. A. (2008). Perception of whether an object affords walking under from different points of observation. Ecological Psychology, 20, 65-83. doi:10.1080/10407410701766650

Warren, W. H., Jr. (1984). Perceiving affordances: visual guidance of stair climbing. Journal of Experimental Psychology: Human Perception E Performance, 10, 683-703. doi.apa.org/journals/xhp/10/5/683.pdf

Warren, W. H., Jr, \& Whang, S. (1987). Visual guidance of walking through apertures: Body-scaled information for affordances. Journal of Experimental Psychology: Human Perception \& Performance, 13, 671-383. doi:10.1037/0096-1523. 13.3.371

Wu, J., He, Z. J., \& Ooi, T. L. (2005). Visually perceived eye level and horizontal midline of the body trunk influenced by optic flow. Perception, 34, 1045-1060. doi:10.1068/p5416

Some recent studies considered single visual stimulation without tilt as the "unimodal" visual condition for testing MLE model on spatial estimates (e.g., Gueguen et al., 2012; Vingerhoets et al., 2009). We adopted the same assumption for studying the integration of visual and body-related cues for judgements of subjective passability. Specifically, we selected the data from two subjects who both ran the three following experiments.

The first experiment corresponded to the now-reported control condition where passability was judged at different body tilts without visual surroundings (body-related cues only: $B$ ).

The second experiment manipulated the optic flow orientation without body tilt; subjects were asked to judge the orientation of the visually induced self-motion relative to the physical horizon (Bourrelly et al., 2010) (visual cues only: $V$ ).

The third experiment manipulated both body-related cues (body tilt) and visual cues (horizontal optic flow) as reported in the present study (body-related cues and visual cues: $B V$ ). 

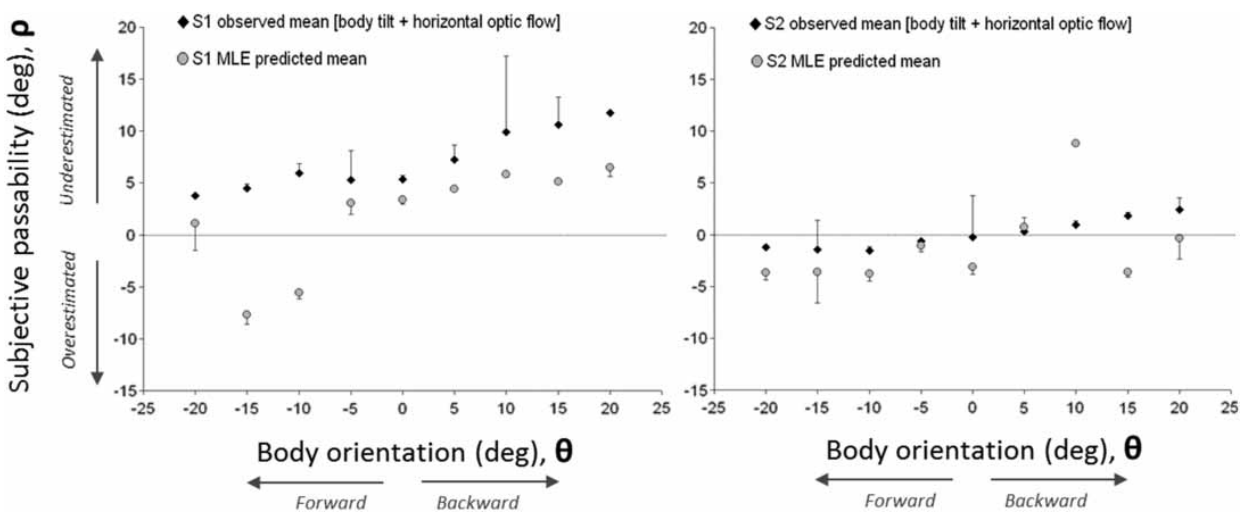

Figure A1. Comparison between observed data and model predictions (Maximum Likelihood Estimations based on the combination of single visual and body-related cues) on judgements of subjective passability relative to the angle of tilt for 2 subjects. Substantial differences were clearly apparent between observed and predicted means for these participants.

We then computed the within-subject reliability of each single condition $(B, V$, and $B V)$ for each angle of tilt. The following equation reflects the computation of the body-related weight $w_{B}$ associated to its reliability according to the measured variance $\sigma^{2}$ observed in $B$ and $V$.

$$
w_{B}=\frac{\sigma_{V}^{2}}{\sigma_{B}^{2}+\sigma_{V}^{2}}
$$

Mean data recorded in both $B$ and $V$ conditions were weighted relative to their reliability to predict data in the combined condition following the equation above:

$$
x_{B V}=w_{B} x_{B}+w_{V} x_{V}
$$

$x_{B V}$ corresponds to the predicted data for the combined condition (i.e., subjective passability at a given angle of body tilt with horizontal optic flow), $x_{B}$ and $x_{V}$ to the mean spatial estimates (i.e., subjective passability or central focus of expansion location) relative to the physical horizon in the respective "unimodal" conditions $B$ and $V$, and $w_{B}$ and $w_{V}$ to their associated reliability. According to MLE, the within-subject variance in the combined condition would depend on the within-subject variance in the unimodal conditions and should be lower. Theoretical withinsubject variance predicted for $B V$ should be as follows:

$$
\sigma_{B V}^{2}=\frac{\sigma_{B}^{2} \sigma_{V}^{2}}{\sigma_{B}^{2}+\sigma_{V}^{2}}
$$

The results of the model for both subjects are illustrated in Figure A1.

As reflected by Figure A1 the predictive power of the present MLE analysis run on two subjects is rather poor for these specific cases. Several interpretations may account for this observation.

First of all, one could argue that the spatial tasks themselves were different between $V$ and $B$ or $B V$ conditions. Indeed, while subjects were asked to judge the direction of the perceived motion induced by optic flow in the $V$ condition, they were asked to judge the capability of passing under an obstacle in the $B$ and $B V$ conditions. Nevertheless, it is worth mentioning that both tasks were geocentric-that is, both types of judgements were referred to the physical horizon and, therefore, might be based on the same underlying processes (as it was already demonstrated for judgements of the gravity-referenced eye level and subjective passability (Bringoux et al., 2008).

Second, one of the major problems when applying this model to spatial perception is that one cannot strictly consider a purely unimodal condition arising from the visual stimulation, since body-related cues can never be suppressed (except for a somatosensory-deafferented patient, who would be also labyrinthine defective!). In other words, the assumption of unimodality in the "single" $V$ condition may be criticized as body-related cues cannot be excluded from the stimulation. As a consequence, the presence of body orientation cues in the $V$ condition could bias the reliability of visual cues in the model and consequently yield substantial differences with the observed data.

Finally, other existing models for spatial multisensory integration might have been at work in the present case, such as "winner-takes-all" models (e.g., Bonneh, Cooperman, \& Sagi, 2001) where a particular sensory cue may "overwrite" all the others. For instance, in line with the last type of model, based on sensory capture phenomenon, it has been shown that the perceived distance of self-motion when both visual and body-related cues were present was perceptually closer to that perceived during physical motion only. Of course, we should remain cautious on these findings and the former interpretations as only two subjects could have been tested from our database. At the same time, only three repetitions per angle/condition were available for intrasubject variance computation. All in one, further investigations remain to be done for improving the predictive power of sensory integration models for spatial orientation.

\section{THE QUARTERLY JOURNAL OF EXPERIMENTAL PSYCHOLOGY, 2014}

